\title{
8
}

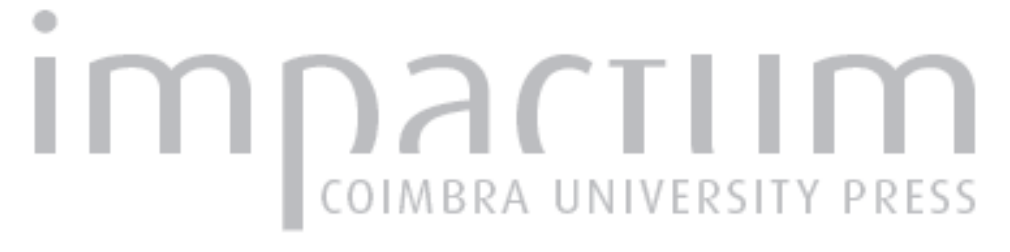

\section{Homère dans les quaestiones convivales}

Autor(es): $\quad$ Ferreira, José Ribeiro

Publicado por: International Plutarch Society

URL persistente:

URI:http://hdl.handle.net/10316.2/37658

DOI:

DOI:http://dx.doi.org/10.14195/0258-655X_8_7

Accessed : $\quad$ 26-Apr-2023 09:40:12

A navegação consulta e descarregamento dos títulos inseridos nas Bibliotecas Digitais UC Digitalis, UC Pombalina e UC Impactum, pressupõem a aceitação plena e sem reservas dos Termos e Condições de Uso destas Bibliotecas Digitais, disponíveis em https://digitalis.uc.pt/pt-pt/termos.

Conforme exposto nos referidos Termos e Condições de Uso, o descarregamento de títulos de acesso restrito requer uma licença válida de autorização devendo o utilizador aceder ao(s) documento(s) a partir de um endereço de IP da instituição detentora da supramencionada licença.

Ao utilizador é apenas permitido o descarregamento para uso pessoal, pelo que o emprego do(s) título(s) descarregado(s) para outro fim, designadamente comercial, carece de autorização do respetivo autor ou editor da obra.

Na medida em que todas as obras da UC Digitalis se encontram protegidas pelo Código do Direito de Autor e Direitos Conexos e demais legislação aplicável, toda a cópia, parcial ou total, deste documento, nos casos em que é legalmente admitida, deverá conter ou fazer-se acompanhar por este aviso.

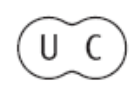




\title{
Homère dans les Quaestiones convivales par \\ José Ribeiro Ferreira \\ Université de Coimbra \\ rifer@fl.uc.pt
}

\begin{abstract}
Homer is frequently mentioned in the Quaestiones Convivales, both explicitly and implicitly. The aim of this study is to analyse these instances or allusions not comprehensively, since they number almost one hundred - and to verify the degree of fidelity in the offered quotations and interpretations of the Homeric text; I try also to understand, if possible, the purposes to which these quotations are due. On acccount of the extensive corpus, I will limit myself to little more than notes and examples. But they will let us to conclude that the references to the Iliad and the Odyssey, and the quotations of lines from these poems, appear in varied contexts and situations, illustrating different types of statement and endorsing opinions, even in insignificant and pointless questions.
\end{abstract}

Key-Words: Homer, Quaestiones Convivales, Banquet, Quotations.

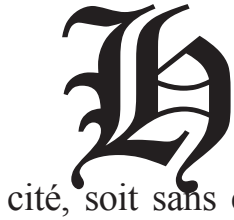

omère est une présence fréquente dans les Quaestiones Convivales, soit explicitement de cette étude est d'analyser ces occurrences ou allusions - certainement pas dans sa totalité, une fois qu'elles parviennent à une centaine - et d'essayer de vérifier le degré de fidélité des citations du texte homérique et sa respective interprétation; finalement, de découvrir les possibles objectifs de son utilisation. A la vue de l'extension du corpus, c'est évident que je n'aborderai que des annotations et ses respectifs exemples.

Homère est possiblement l'auteur plus utilisé par Plutarque, environ 1300 citations et références, si on inclut aussi celles qui apparaissent dans $D e$ Homero, d'auteur controversé ${ }^{1}$. En ce

Vide G. D' Ippolito, “L' Omero di Plutarco", in Italo Gallo (ed.), La Biblioteca di Plutarco. Atti del IX Convegno plutarcheo, Pavia, 13-15 giugno 2002 (Napoli, 2004), p. 15-16. Pour les citations homériques dans les Quaestiones Convivales je suis, normalement, la classification et la distinction établies dans cette étude de G. D’Ippolito, avec une ou deux adaptations. 
qui concerne le traitement du texte homérique par Plutarque, on les divise en compendieuses ou paraphrastiques - quand elles s'éloignent de l'original et ne présentent qu'un résumé de tout le contenu ou juste d'une partie- et en littéraires, les vraies citations, quand elles reproduisent le texte original, si bien qu'on puisse introduire des altérations et des adaptations, involontaires ou volontaires. En ce qui concerne l'objectif, la fonction primaire des citations homériques (et celles des poètes en général) en Plutarque est celle de témoignage et autorité, si bien qu'il y en ait d'autres, comme la fonction politique et paideutique (il faut se rappeler du valeur politique et paideutique qui exerce l'œuvre de Homère et du rôle de paradigmes de plusieurs de ses héros), l'esthétique ou décorative, la ludique, l'étique (la citation de sentences ou gnomai comme expression des valeurs morales) et même encore la fonction scientifique.

Les Quaestiones Convivales confirment la tendance générale de l'œuvre de Plutarque, dans le numéro de citations homériques, dans ses fonctions et même dans le traitement du texte. L'Iliade et l'Odyssée ne sont pas seulement à l'origine du thème de plusieurs conversations - sept dans son ensemble (nommément II. 5; V. 4, 8 et 10; VI. 9; et IX. 4 et 13) - mais elles offrent aussi des constantes citations de vers et expressions, des paraphrases des épisodes, ou même des pures allusions, pour supporter des conversations ou pour justifier des affirmations. Par exemple, l'éloge de Platon (717E) est souligné par un passage de l'Iliade (XXIV.258-259), auquel Priam dit de Hector qu' «il ne semblait pas fils d'un mortel mais plutôt d'un dieu » (oú-

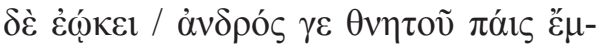

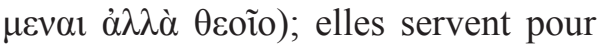
rapprocher un problème juridique (741D); elles montrent que les sirènes homériques étaient effrayantes (745D). Son témoignage est introduit comme autorité dans la discussion sur la raison par laquelle les Pythagoriques ne mangent pas du poisson, une fois que on argumente - dans l'Iliade et dans 1'Odyssée, les Achéens et les Phéaciens n'en mangent pas non plus (730C).

Je n'ai pas l'intention, et ça ne me paraît pas convenable, de continuer avec cette énumération de références et citations parce que d'abord le temps ne le permet pas mais aussi parce qu'elle deviendrait longue et ennuyante. Je me permettrais seulement de donner encore deux exemples qui me paraissent appropriés aux conversations pendant les banquets. Le premier, dans 617E, se rapporte à une citation d'un passage de l'Iliade (XXIII. 534 sqq.) dans laquelle Achille, en voyant Ménélas et Antiloque en disputant la deuxième place dans une course à cheval, en craignant leur rivalité, concède le prix à Eumèlos. C'est une référence qui apparaît en soutenant le besoin d'une disposition sensée entre les conviviales 
au banquet, sans la lâcher au libre choix, au contraire, prenant soin de toutes les fois qu'il y ait des invités avec des mérites semblables ou bien avec une rivalité entre eux - donner la distinction à l'autrui (le père, le grandpère, un familier proche).

Le deuxième exemple (645A), Homère, appelé seulement « le poète », d'une part, soutien que le partage de la table aide à connaître les hommes et que le vin révèle son caractère; de l'autre, avec la citation de trois vers du Chant XIV de l'Odyssée (vv. 464-466), il justifie la distinction entre la joie, provoquée par le boisson qui fait chanter, rire, danser et amène au commerce sain - et l'ivresse qui cause le déréglage et qui conduit au bavardage et à

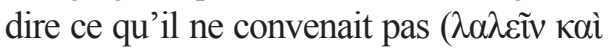
$\lambda \varepsilon ́ \gamma \varepsilon เ v \tilde{\alpha} \beta \varepsilon \dot{\varepsilon} \lambda \tau \iota v v \tilde{\eta} v \sigma \omega \pi \tilde{\alpha} v)$.

Ayant déjà été étudiées les paraphrases et les citations cachées (allusions, imitations, remissions) par Juan Manuel Díaz Lavado ${ }^{2}$, je m'occuperai des citations vraies ou littéraires qui - constituées par un seul mot, par un hémistiche, ou bien par un ou plusieurs vers - peuvent apparaître d'une façon cachée sans aucun signal de présentation (il s'agit de ce qu'on appelle les citations anonymes) ou bien d'une façon explicite, avec un lemme ou un contexte de présentation, avec une mention, ou pas, de son auteur, signalée quelques fois

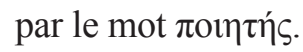

Si la référence explicite à Homère ou à l'Iliade et à l'Odyssée est la situation la plus commune, quelques fois, ni l'un ni les autres apparaissent nommés (environ vingt-cinq fois) en espérant - c'est évident - que le public ou bien les interlocuteurs connaissaient celui qui était considéré le poète par excellence. C'est peut-être à cause de cela que, souvent, il apparait comme un « homme habile » (615E) ou juste simplement comme "le poète' (c'est le cas de la dernière citation), soit à cause du fait que le texte et l'auteur soient connus, soit parce qu'il y a des informations supplémentaires qui permettent l'identification ${ }^{3}$. C'est ce qui arrive en $624 \mathrm{E}$ et $655 \mathrm{~A}$ - pour donner seulement deux exemples parmi

"Paráfrasis homéricas en Plutarco", in J. A. Fernández Delgado y Francisca Pordomingo PARDO (edd.), Estudios sobre Plutarco: Aspectos formales. Actas del IV Simposio Español sobre Plutarco, Salamanca - 26 a 28 de Mayo de 1994 (Madrid, 1996), pp. 429-445; "Discutiendo sobre Homero en torno a una copa de vino: Los zetemata homérica en el marco del banquete", in J. G. Montes Cala, M. Sánches Ortiz de Landaluce y R. J. Gallé Cejudo (edd.), Plutarco, Dioniso y el vino. Actas del VI Simposio Español sobre Plutarco, Cádiz, 14-16 de Mayo de 1998 (Madrid, 1999), pp. 199-209; et Las citas de Homero en Plutarco, Zaragoza, 2010.

3 Vide G. D’ Ippolito, “L' Omero di Plutarco”, in Italo Gallo (ed.), La Biblioteca di Plutarco. Atti del IX Convegno plutarcheo, Pavia, 13-15 giugno 2002 (Napoli, 2004), p. 18. 
la douzaine d'occurrences. Au premier exemple - inclus dans la Question $6 \mathrm{du}$ Livre I qui traite la propension d'Alexandre à boire (623D-624F) presque trois vers de l'Iliade (IX. 846848) sont cités et ils nous parlent de la cicatrisation des blessures avec des médicaments amères, passage présenté seulement avec l'éclaircissante expression "comme dit le poète » ( $\dot{\omega} \varsigma$

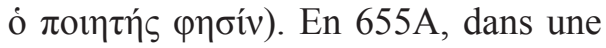
conversation sur le meilleur moment pour l'amour (III. 6), on trouve une nouvelle référence à Homère - de nouveau nommé simplement par ó $\pi$ ointńs; il s'agit de l'épisode do Chant III de l'Iliade où Pâris est retiré du combat (vv. 428-447) après avoir perdu le duel avec Ménélas et retourne à son logement auprès d'Hélène un passage qui non seulement a une critique implicite envers l'attitude du prince troyen mais a aussi produit plusieurs commentaires à travers les temps, comme réfère d'ailleurs le scoliaste dans le commentaire au vers 441. Ce n'est pas donc surprenant un commentaire moral des Quaestiones Convivales et qu'il soit répété dans De audiendis Poetis (18F). Je vous transcris le texte de Plutarque :

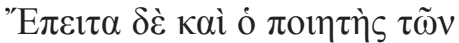

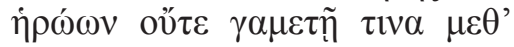

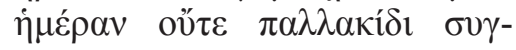

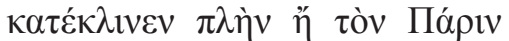

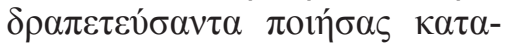

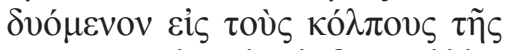

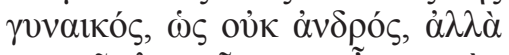

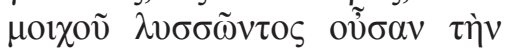

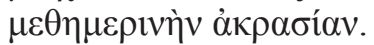

Je vous citerai aussi le Poète: aucun de ses héros ne se couche durant la journée ni avec son épouse ni avec sa maîtresse; s'il a représenté Pâris se réfugiant, après avoir fui la bataille, dans les bras de sa femme, cela signifie que l'intempérance, en plein jour, est le fait, non d'un époux, mais d'un adultère enragé $e^{4}$.

Homère apparaît, ainsi, comme le poète par excellence, ça veut dire, le Poète. Il est, d'ailleurs, significativement

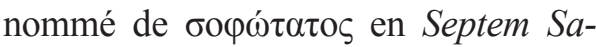
pientium Convivium (164D).

Ce n'est pas donc surprenant que, dans les Quaestiones Convivales, la fonction primaire des citations homériques (et celle des poètes en général) - si bien que d'autres puissent exister, comme l'esthétique, l'étique, la politique, la paideutique, la scientifique soit celle du témoignage, de l'autorité. C'est pourquoi, des termes des Poèmes Homériques appellent le sel de "divin" ( $\theta \varepsilon$ ĩov: 684F e 697D) ${ }^{5}$ et un passage du Chant XI de l'Iliade (vv. 7-8), mentionne que les navires de Ajax étaient proches de ceux d'Achille (679B).

4 Traduction de Fr. Funrmann, Plutarque, Oeuvres Morales. IX, Première Partie (Paris, 1972), p. 134.

5 Citation de $I l$. XI. 7-8 et IX. 214, respectivement. 
Je me permets de souligner aussi trois passages qui exemplifient la garantie scientifique des citations homériques. Dans la Question I. 9, le rendez-vous d'Ulysse avec Nausicaa (Od. VI 59 e 226), où la jeune princesse fait le linge dans la rivière et pas dans la mer qui était beaucoup plus proche(627A) et où Ulysse apparaît dans un état lamentable après le naufrage (627E-F), ces deux situations soutiennent la conversation qui essaie de démontrer qu'on fait mieux le linge dans l'eau de la rivière que dans l'eau de la mer; et dans ce passage, Homère dépasse même l'autorité d'Aristote. Dans la Question 10 et dernière du Livre III (657F-659D), la discussion sur la détérioration plus rapide de la viande sous les effets de la lune et l'affirmation que les rayons solaires sèchent mieux que ceux de la lune, cela nous apporte (658B) au passage de l'Iliade où Apollon allonge une nuage sur le corps de Hector pour empêcher que les rayons de soleil le séchaient en excès (XXIII. 190-191).

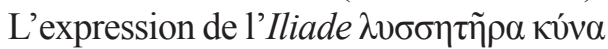
'chien enragé' (VIII. 299) est introduite, en parlant de lèpre et d'hydrophobie, pour démontrer que Homère connaissait déjà ces maladies (732A).

En quelques passages, les citations apparaissent même déviées de leur sens propre et sont interprétées hors de leur contexte. Prenons deux exemples.

Le premier, c'est dans la Question 5 du Livre II - basée en entier dans l'épisode des jeux funèbres en honneur de Patrocle (Iliade XXIII, 262-897) - où des passages des compétitions funèbres en honneur de Patrocle (Il. XXIII. 620-623 e 634-636) et des jeux organisés par Alcinoos pour Ulysse (Od. VIII. 206 e 246-247) sont introduits pour soutenir la séquence que les épreuves athlétiques (639A-640A) doivent suivre et où on discute la raison par laquelle Homère place en premier l'épreuve de pugilat, ensuite la lutte et en dernier la course (639A-640A). La question est abordée de la manière suivante, peut-être par Timon ${ }^{6}$ (639B):

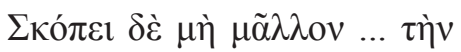

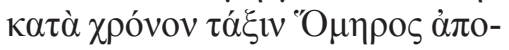

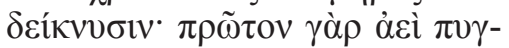

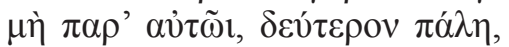

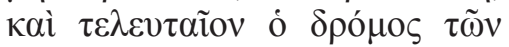

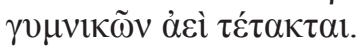

On peut se demander, dit-il, si l'ordre chronologique n'est pas mieux indiqué par Homère; les concours gymniques sont toujours, chez lui, ordonnés de la même façon: en premier lieu le pugilat, en second lieu la lutte, et en dernier la course ${ }^{7}$.

6 Le texte transmit par les manuscrits n'est pas, sûrement, complet. Une partie d'une parole de Timon s'est peut-être perdue. Vide Fr. Fuhrmann, Plutarque, Oeuvres Morales. IX, Première Partie (Paris, 1972) I-III, p. 184, nota ad locum).

7 Traduction de Fr. Fuhrmann, Plutarque, Oeuvres Morales. IX, Première Partie (Paris, 1972), p. 85.

Ploutarchos, n.s., 8 (2010/2011) 81-90

ISSN 0258-655X 
Timon poursuit en disant que les jeux funèbres en honneur de Patrocle ont la séquence indiquée, semblant négliger que les jeux funèbres commencent avec l'épreuve plus importante et plus longuement décrite, la course de voiture à cheval (vv. 262619), et introduisant, au contraire, des passages où les épreuves apparaîtraient nommées toujours par la séquence déjà présentée $^{8}$ : Achille dit à Nestor (vv. 620-623) que, à cause de sa vieillesse, il lui donnera un prix par déférence, sans avoir besoin de faire ni le pugilat, ni la lutte, ni la compétition du lancer du javelot, ni la course (639B-C). L'ancien lui répond (vv. 634-636) qu'il a déjà gagné les épreuves mentionnées, et par la même séquence (639C). Ensuite, il fait appel à des passages de la narration des jeux organisés par les Phéaciens en honneur d'Ulysse, dans le Chant VIII de l'Odyssée, où Céphale (v. 206) et Alcinoos (vv. 246247) indiquent les épreuves dans la séquence citée (639C-D). C'est curieux, et surprenant au même temps, d'observer que Plutarque, dans sa première citation, non seulement il ignore la longue et déjà mentionnée course de voiture, mais il oublie aussi l'épreuve du lancer du javelot, placée entre la lutte et la course, et change les mots de Nestor (vv. 634-636), éliminant la référence faite dans les deux vers suivants au lancer du javelot et à la course à cheval (vv. 637-638).

Le deuxième exemple, je le trouve dans la Question 4 du Livre V (677C678B), toute une discussion sur l'ordre de Achille à Patrocle dans le Chant IX de l'Iliade (vv.203-204) pour qu'il

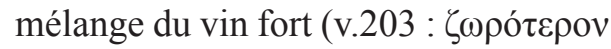
$\delta \varepsilon \grave{\varepsilon} \kappa \varepsilon \alpha \iota \varepsilon$ «mélange du vin bien fort») dans un grand kratêr ${ }^{9}$. Et Péléide justifie ainsi son ordre : « des amis très estimés sont accueillis sous mon toit »

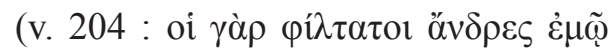

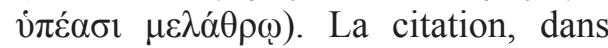
ce passage de Quaestiones Convivales, apparaît un peu déviée de son sens, même hors contexte, à un point qu'elle change le sens original du texte homérique, car la paraphrase interprète 1'expression comparative $\zeta \omega$ -

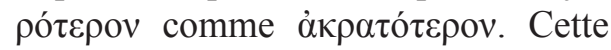
comparaison motive tout de suite une

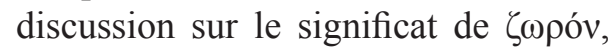
sur lequel, une fois que ce n'appartient pas à mon objectif, j'en parlerai juste un peu. Tandis que Nicératos de $\mathrm{Ma}$ -

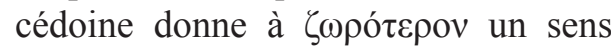
de "bien mélangé », Plutarque défends le significat de " plus pur », étant en désaccord avec ceux, comme Zoïle de

8 Sven-Tage Teodorson, A Commentary on Plutarch's Table Talks, vol. I, Books 1-3 (Göteborg, 1989), p. 241 ad 639B.

9 L'habitude de mélanger de l'eau avec du vin était très ancienne chez les Grecs ; vide les volets en Linear B. Vide B. Hainsworth, The Iliad A Commentary III: books 9-12 (Cambridge, 1993), p. 90 ad. 11. 202-204; J. Griffin, Home: Iliad IX, edited with an introduction and commentary (Oxford, 1995), p. 100 ad 1. 203. 


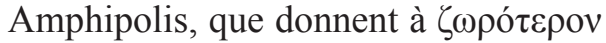
le sens de «plus chaud» a fin d'éviter mettre une inconvenance sur la bouche d'Achille. Il défend, en faveur du Péléide, et comme justification de sa séquence, d'un côté, le fait que Phénix et Ulysse soient des hommes plus âgés qui préfèrent le vin avec moins d'eau

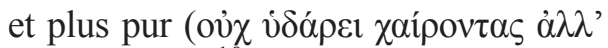
$\dot{\alpha} \kappa \rho \alpha \tau o ́ \tau \varepsilon \rho \circ)^{10}$, et d'autre côté, le fait que Achille ait appris chez Quirinus que les hommes épuisés par une journée entière de lutte demandent un traitement différent de ceux que ne font rien et à qui on doit donner un mélange plus faible.

Cette discussion sur le sens de $\zeta \omega$ -

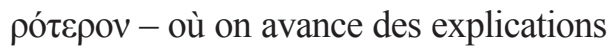
étymologiques, quelquesunes fantaisistes, comme en 677C et 677D-E - utilise des citations d'autres plusieurs auteurs grecs et invoque l'éducation d'Achille chez Quirinus et les connaissances diététiques acquises qui l'ont permis de savoir que le corps humain en repos doit avoir un régime alimentaire plus léger, au contraire de ceux qui passent la journée à combattre $(677 \mathrm{~F}-678 \mathrm{~B})$. Il termine la question en affirmant que Achille n'était pas, par nature, quelqu'un qui

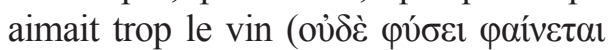
pírotvos), mais il avait un tempérament

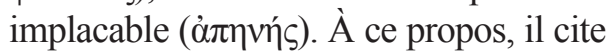

les vv. 467-468 du Chant XX d'Iliade qui ne le classifient pas comme doux et facile mais plutôt comme un homme

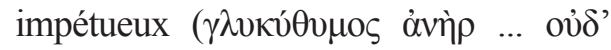

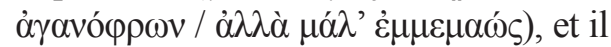
cite encore une partie du v. 325 du Chant IX qui parle de ne pas fermer l'œil de la

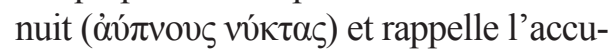
sation de oivoßapท́s que le héros lance à Agamemnon (Il.I. 225).

En quelques passages, les citations ne suivent pas exactement le texte homérique ni transcrirent non plus une leçon qui n'est pas la plus acceptée. Si la plupart des fois elles sont précises et les coïncidences avec le texte homérique des meilleures éditions modernes se vérifient dans une proportion remarquable ${ }^{11}$, il y en a aussi des variantes, soit de flexion, soit d'omission ou d'addition, soit de transposition ou de substitution des mots - ces variations peuvent être involontaires ou bien volontaires, causées par des erreurs de mémoire ou par l'adaptation au contexte. On trouve un exemple d'une variante, à cause d'un choix d'une leçon différente, en 675A, où - après avoir fait allusion au concours entre Homère et Hésiode - il parle des jeux funèbres en honneur de Patrocle et des prix d'éloquence que Achille

10 La préférence des hommes plus âgés par le vin plus for et pur occupe toute la discussion de la Question 7 du Livre I et aussi abordée dans la Question 3 du Livre III (650D-E).

11 G. D’ Ippolito, «L' Omero di Plutarco», in Italo Gallo (ed.), La Biblioteca di Plutarco. Atti del IX Convegno plutarcheo, Pavia, 13-15 giugno 2002 (Napoli, 2004), p.31 parle en $80 \%$ de coïncidences, en citant même une thèse non publiée, défendue à Liège en 1936, par M. Boulanger, Les citations de L'Iliade dans la Vita Homeri attribuée à Plutarque. 


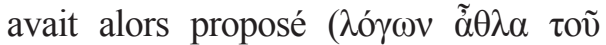
A $\chi 1 \lambda \lambda \varepsilon \dot{\varepsilon} \omega \varsigma \pi \rho \circ \theta \varepsilon \dot{v} \tau 0 \varsigma)$, préférant, pour Il. XXIII. 886, une leçon du texte ho-

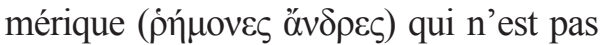

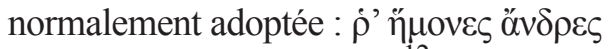
(«les lanceurs de javelots») ${ }^{12}$. D'ailleurs, les Quaestiones Convivales elles même spécifient qu'il y a une référence à des prix pour les orateurs si on adopte la leçon $\rho \eta ́ \mu o v \varepsilon \varsigma$ et pas $\eta \mu \mathrm{ov \varepsilon \varsigma} \mathrm{:}$

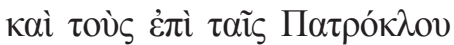

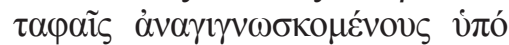

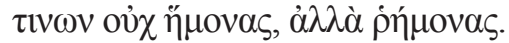

En 714A-B, la question d'être autant grecque que perse cette tradition de faire des délibérations pendant les banquets (714A-C) apparaît illustrée avec des passages de l'Iliade et de l'Odyssée. Dans le premier poème, on prend l'épisode du Chant IX où les chefs Achéens, réunis dans la tente d'Agamemnon, décident d'envoyer une ambassade à Achille et sont cités des vers de la parole de Nestor. Je transcris le passage où on trouve les citations d'Il. IX. 93, 70 et 74-75, respectivement :

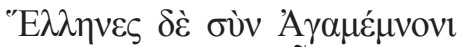

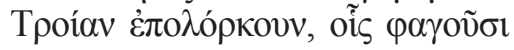

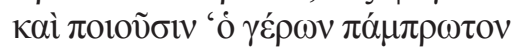

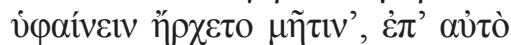

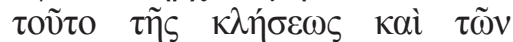

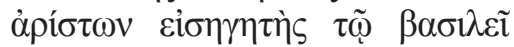

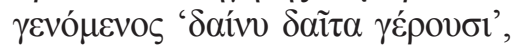

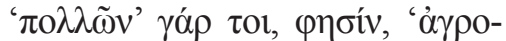

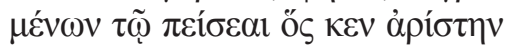

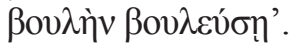

C'est devant des Grecs qui assiégeaient Troie avec Agamemnon que, alors qu'ils avaient mangé et bu, «le vieillard commença à tisser les fils de son projet» en proposant au roi d'inviter les chefs à cette fin précisément: «donne un dîner aux anciens» et «justement, dit-il, au sein de cette nombreuse assemblée, suis celui qui donnera le meilleur avis» ${ }^{13}$.

Peu après, dans la même question (714C), les Quaestiones Convivales rappèlent le passage du Chant VII de l'Odyssée où Ulysse rencontre les Phéaciens en faisant des libations à Hermès (vv. 136-138), comme ils faisaient toujours avant de se coucher ( $\pi \nu \mu \alpha \dot{\alpha} \omega \sigma \pi \varepsilon ́ \delta o v \tau \varepsilon \varsigma$ ö $\tau \varepsilon \mu \nu \eta \sigma \alpha i ́ \alpha \tau o$ кoí-

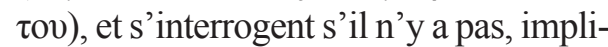
cite, une association du vin à la raison. Les citations présentent, pourtant, une altération du texte original, peut-être pour l'adapter à la syntaxe de la phrase : celle du vers 93 de l'Iliade remplace la forme

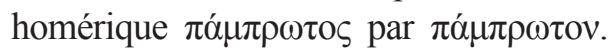
Dans celle de l'Odyssée apparaît le participe $\sigma \pi \varepsilon \dot{\delta} \delta \nu \tau \varepsilon \varsigma$ tandis que dans le texte homérique on trouve $\sigma \pi \varepsilon ́ v \delta \varepsilon \sigma \kappa o v$ (v. 138). C'est convenant aussi d'observer que les libations aux divinités, faites avant que les héros s'aillent coucher

12 Sur le sujet, vide N. Richardson, The Iliad: A Commentary VI. Books 21-24 (ed. G. S. KIRK, Cambridge, 1993) 270 ad lin.

13 Traduction de Françoise Frazier et J. Sirinelli, Plutarque, Oeuvres Morales. IX, Troisième Partie (Paris, 1996), p. 55 
- pratique fréquente dans les Poèmes Homériques - sont normalement faites à Zeus et aux dieux olympiques ${ }^{14}$. Ce passage est la première référence à des libations à Hermès, si bien que, vis-àvis aux circonstances, c'est toujours approprié de les diriger à un dieu qui est en rapport avec le sommeil et les rêves (cf. Il. XXIV. 343-344 e 445; Od. V. 4748 e XXIV. 1-4). Ce geste, ne sera t'il pas une certaine manière des Phéaciens d'obtenir un sommeil réparateur et des bonnes rêves?

Un autre cas de variante textuelle, on la trouve dans la conversation sur la danse et ses mouvements (IX. 15, 747DE) où on cite deux vers du Chant XXIII de l'Iliade (vv. 503-504), comme capacité poétique du poète de représenter, d'une manière vivante, la course de voiture à cheval. Les Quaestiones Convivales, toutefois, nous donnent le syntagme $\delta$ ' $\alpha \tilde{v} \chi \alpha \lambda \kappa \tilde{\omega}$, à la fois de $\delta \dot{\varepsilon} \chi \rho v \sigma \omega \tilde{~ d u ~ t e x t e ~}$ homérique, peut-être parce qu'il cite de mémoire qul l'a trahit et lui a fourni l'expression plus fréquente en Homère (125 occurrences contre seulement 8$)^{15}$.

En conclusion:

Les références à l'Iliade et à l' Odyssée, les citations de leurs vers, apparaissent dans les plus divers contextes et situations, illustrent des différentes affirmations, corroborent des opinions, même s'il s'agit des questions insignifiantes et futiles: par exemple, elles illustrent - Iliade III. 375, qui se rapporte à un boucle fait de peau d'un veau abattu - l'affirmation que la peau des animaux souffre des modifications selon la mort qu'ils ont (642E); elles supportent aussi la démonstration que les animaux sont plus exposés que les hommes aux maladies contagieuses (662E); et elles sont encore le point de départ pour la conversation qui discute quelle est la main d'Aphrodite blessée par Diomède (739B).

$\mathrm{Si}$, parfois, Homère - dont on fait allusion à sa légendaire compétition poétique avec Hésiode en $675 \mathrm{~A}$ - est référé d'une manière négative, comme par exemple l'accusation de rien connaître sur l'amour (618D) et la critique à ses banquets (643D), il apparait, normalement, une autorité et référence crédibles.

Les divergences et les changements qu'on trouve par rapport au texte homérique peuvent advenir d'une tradition textuelle différente, une erreur de mémoire ou une adaptation à un nouveau contexte.

14 Vide A. F. Garvie, Homer, Odyssey. Books VI-VIII (Cambridge, 1994), p. 192 ad. 11. 136-138.

15 Vide Sven-Tage Teodorson, A Commentary on Plutarch's Table Talks, vol. III, Books 7-9 (Göteborg, 1996), p. 381; G. D’ Ippolito, «L' Omero di Plutarco», in Italo Gallo (ed.), La Biblioteca di Plutarco. Atti del IX Convegno plutarcheo, Pavia, 13-15 giugno 2002 (Napoli, 2004), p. 20. 